\title{
MIXED SELFING AND RANDOM MATING WITH POLYSOMIC INHERITANCE
}

\author{
GILLIAN McCONNELL and J. L. FYFE \\ Scottish Plant Breeding Station, Pentlandfield, Roslin, Midlothian
}

Received 8.xi.74

\begin{abstract}
SUMmary
Given a large population of a $2 k$-ploid organism, reproducing by constant proportions of selfing $(s)$ and random mating $(1-s)$, with no double reduction and no selection, the theoretical population inbreeding coefficient $(F)$ at equilibrium is $s /\{2 k-(2 k-1) s\}$.
\end{abstract}

\section{INTRODUCTION}

IT is well known that a diploid population with constant rates of selfing $(s)$ and random mating $(1-s)$ and no selection comes to a theoretical equilibrium with $F$, the population inbreeding coefficient, equal to $s /(2-s)$. There does not appear to be any published generalisation to polyploids, although some 20 years ago A. R. G. Owen gave one of us (J. L. F.) the corresponding result for a tetraploid with no double reduction: $F=s /(4-3 s)$. As applied to polyploids, $F$ measures the probability that two alleles, chosen at random at one locus in one randomly chosen individual, will be identical by descent from a single allele in some ancestor.

\section{THEORY}

Self-fertilisation of a $2 k$-ploid organism, with no double reduction, leads to the recurrence relation (Haldane, 1930; Wright, 1938; Kempthorne, 1957):

$$
2(2 k-1) F_{n+1}=1+(4 k-3) F_{n} .
$$

Random mating in a population large enough to preclude mating of relatives gives:

$$
(2 k-1) F_{n+1}=(k-1) F_{n} .
$$

At equilibrium, with a proportion of $s$ selfing and $(1-s)$ random mating, constant over generations:

$$
2(2 k-1) F=s+s(4 k-3) F+2(1-s)(k-1) F
$$

leading to $F=s /\{2 k-(2 k-1) s\}$.

With $k=1$ and $k=2$ this gives the expressions already quoted for diploids and tetraploids respectively; hexaploids $(k=3)$ give $F=s /(6-5 s)$, octaploids $(k=4), F=s /(8-7 s)$ and so on. 


\section{REFERENGES}

HALDANE, J. B. s. 1930. Theoretical genetics of autopolyploids. 7. Genet., 22, 359-372. KEMPTHORNE, O. 1957. An introduction to genetic statistics, p. 93. Wiley, New York. WRIGHT, s. 1938. The distribution of gene frequencies in populations of polyploids. Proc. nat. Acad. Sci. Wash., 24, 372-377. 